\title{
Short-term wind power prediction based on extreme learning machine with error correction
}

\author{
Zhi Li ${ }^{1}$, Lin Ye ${ }^{1 *}$, Yongning Zhao ${ }^{1}$, Xuri Song ${ }^{2}$, Jingzhu Teng ${ }^{1}$ and Jingxin Jin $^{3}$
}

\begin{abstract}
Introduction: Large-scale integration of wind generation brings great challenges to the secure operation of the power systems due to the intermittence nature of wind. The fluctuation of the wind generation has a great impact on the unit commitment. Thus accurate wind power forecasting plays a key role in dealing with the challenges of power system operation under uncertainties in an economical and technical way.

Methods: In this paper, a combined approach based on Extreme Learning Machine (ELM) and an error correction model is proposed to predict wind power in the short-term time scale. Firstly an ELM is utilized to forecast the short-term wind power. Then the ultra-short-term wind power forecasting is acquired based on processing the short-term forecasting error by persistence method.

Results: For short-term forecasting, the Extreme Learning Machine (ELM) doesn't perform well. The overall NRMSE (Normalized Root Mean Square Error) of forecasting results for 66 days is $21.09 \%$. For the ultra-short term forecasting after error correction, most of forecasting errors lie in the interval of [-10 MW, $10 \mathrm{MW}]$. The error distribution is concentrated and almost unbiased. The overall NRMSE is $5.76 \%$.

Conclusion: The ultra-short-term wind power forecasting accuracy is further improved by using error correction in terms of normalized root mean squared error (NRMSE).
\end{abstract}

Keywords: Ultra-short-term forecasting, Wind power forecasting, Extreme Learning Machine, Error correction

\section{Introduction}

Wind power has been undergoing a rapid development in recent years. The large-scale integration of wind power is challenging power grids operation and management [1]. Compared with conventional generation, one of the largest problems of wind power is its dependence on the volatility of the wind [2]. Unexpected variations of wind generation may increase operating costs, increasing reserves requirements, and posing potential risks to system reliability. In order to schedule the spinning reserve capacity and manage the grid operation, persistence approach was commonly used to predict changes of the wind power production in the ultra-short-term [3, 4].

\footnotetext{
* Correspondence: yelin@cau.edu.cn

${ }^{1}$ College of Information and Electrical Engineering, China Agricultural University, P. O. Box 210, Beijing 100083, Peoples Republic of China Full list of author information is available at the end of the article
}

Wind power is impacted by wind speed, temperature, humidity, latitude, terrain topography, air pressure, and other factors [5]. Modeling the behavior of wind is a challenge due to its stochastic nature. The existing forecasting methods can be mainly classified as physical approaches, statistical approaches, as well as a combination of both [6]. The physical models use physical considerations, such as meteorological (numerical weather predictions), and topological (orography, roughness, obstacles) information and technical characteristics of the wind turbines (hub height, power curve, thrust coefficient) [7]. Statistical models find the relationships between a set of explanatory variables including NWP results and online measured generation data [7]. The basic statistical approach includes the time-series analysis and neural networks, such as Box-Jenkins ARMA $(p, q)$ models, where $p$ represents most recent wind speeds and $q$ represents most recent forecasting errors [8]. Neural network $(\mathrm{NN})$ models have been widely applied in a variety 
of business fields including accounting, management information systems, marketing, and production management. Many researchers focus on the improvement of $\mathrm{NN}$, including recurrent NN, deep NN and so on [9-14]. Extreme Learning Machine(ELM) is based on a single-hidden layer feed-forward neutral network and only needs to calculate random weight between inputting layer and hidden layer. The performance of ELM is better than traditional NN in terms of numerical experiments. Furthermore, the combined models have been widely used to improve wind power forecasting accuracy. Wind power forecasting method based on empirical mode decomposition (EMD) and support vector machine (SVM) was proposed to cope with the nonlinearity and non-stationarity of wind speed data. The combined approach can improve the forecasting accuracy by $5-10 \%$ compared to single statistics [15].

Several wind power forecasting tools have been developed across the world. A Wind Power Prediction Tool (WPPT) is developed to predict the wind power on various time scales, from half an hour to $36 \mathrm{~h}$ ahead. This tool is based on adaptive recursive least square estimation with exponential forgetting factor [16]. The WPPT can forecast the wind power production in relatively large geographical regions. For each individual wind farm, it uses statistical models to describe the relationship between observed power production and the weather predictions. Another tool named as the Prediktor developed at Risø mainly uses physical relations to transform the predicted wind into predicted power [17]. The Zephyr tool is the combination of the Wind Power Prediction Tool (WPPT) and Prediktor tool and its main goal is to merge Prediktor and WPPT to obtain synergy between the physical and the statistical approach [17]. The Sipreolico tool, developed by the University of Carlos III of Madrid, consists of nine adaptive nonparametric statistical models that are recursively estimated with either the recursive least squares algorithm or a Kalman filter. The tool is based on Spanish HIRLAM forecasts, taking into account hourly SCADA data from $80 \%$ of all Spanish wind turbines [18]. The EWIND model developed by TrueWind, Inc. applies a once-and-for-all parameterization for the local effect by using the output of the ForeWind NWP model, and it uses either a multiple screening linear regression model or a Bayesian neural network to find out the systematic errors [19]. The Advanced Wind Power Prediction tool (AWPT) was developed by ISET (the institute of "Solare Energieversorgungstechnik") and the tool uses weather forecasts coming from Lokalmodell (LM) of the Deutsche Wetterdienst (DWD) and predicts the wind power with artificial neural networks [20]. Ecole de Mines de Paris (ARMINES) and Rutherford Appleton Laboratory (RAL) have developed models for shortterm prediction based on fuzzy-neural networks [20].

The individual forecasting method cannot achieve a high accuracy due to the intrinsic characteristics of wind speed and wind power. In this paper, a combined statistical approach for wind power forecasting is presented by using Extreme learning method and error correction. The ultra-short-term power forecasting is acquired based on processing the forecasting error of short-term forecasting results based on the persistence method in terms of Normalized Root Mean Square Error (RMSE).

\section{Methods}

\section{Extreme Learning Machine (ELM)}

ELM theory was proposed to predict wind power, which tends to provide good generation and performance at extremely fast learning speed in theory and practical applications. ELM has the following several advantages:

1) The parameters of ELM can be set easily, and ELM originally can get a good performance only with fitful references in hidden layers.

2) The computation of ELM is efficient, which does not need as many iterations as Neural Network (NN) and as complexity as Support Vector Machine (SVM) in when solving quadratic optimization.

3) ELM has good generalization performance. And the experimental results show that the ELM can achieve good generalization performance in most cases and can learn faster than feed-forward neural networks [21].

Now the EML has been widely used in several fields such as face recognition, image classification,wind prediction in short-term scale. Wind power forecasting can be regarded as an ELM problem, because some factors such as wind speed, air condition, temperature \& humidity, wind turbine arrangement have influence on wind production. As for how they exactly affect wind production has not been clearly known [22]. ELM model can be established by using example data and predict the curve of power in short-term.

The ELM model is based on a single-hidden layer feed-forward neural network (SLFN). The advantage of the ELM algorithm is that it distributes the weights and thresholds between the inputting layer and the hidden layer in random and does not need to adjust these random parameters during the whole learning process so that it can complete the training process extremely fast. Based the above advantage, ELM is chosen as a predictor to predict day-ahead wind power in the short-term time scale. The structure of a standard ELM network is demonstrated in Fig. 1.

The main parameters of ELM are described as follow:

$$
\boldsymbol{\omega}=\left[\begin{array}{cccc}
\omega_{11} & \omega_{12} & \cdots & \omega_{1 n} \\
\omega_{21} & \omega_{22} & \cdots & \omega_{2 n} \\
\vdots & \vdots & \vdots & \vdots \\
\omega_{l 1} & \omega_{l 2} & \cdots & \omega_{l n}
\end{array}\right]_{l \times n}
$$




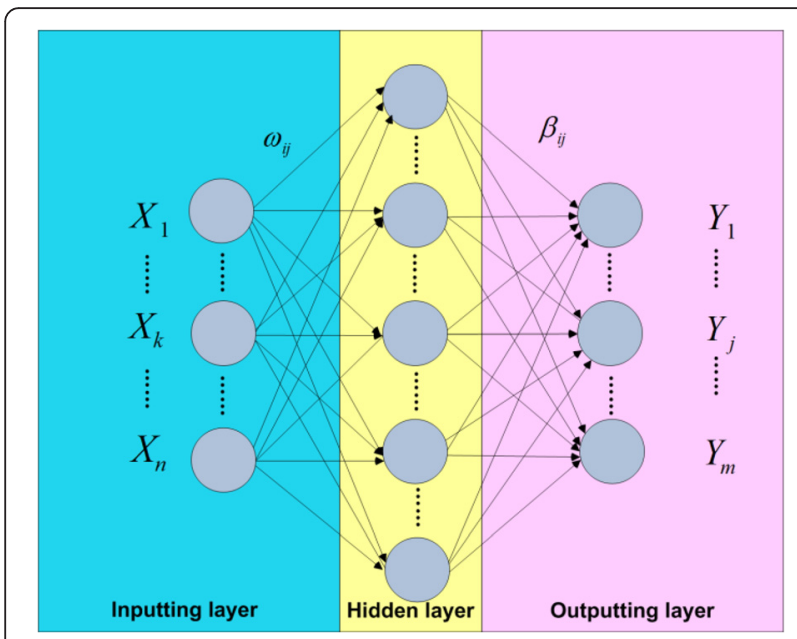

Fig. 1 Architecture of Extreme Learning Machine

where ' $\omega$ ' is the network weight between the input layer and the hidden layer, and ' $\omega_{i j}$ ' is the weight between the $i^{\text {th }}$ input node of the input layer and the $j^{\text {th }}$ hidden node of the hidden layer. ' $l$ ' is the number of input nodes in input layer. ' $n$ ' is the number of hidden nodes in output layer.

$$
\boldsymbol{\beta}=\left[\begin{array}{cccc}
\beta_{11} & \beta_{12} & \cdots & \beta_{1 m} \\
\beta_{21} & \beta_{22} & \cdots & \beta_{2 m} \\
\vdots & \vdots & \vdots & \vdots \\
\beta_{l 1} & \beta_{l 2} & \cdots & \beta_{l m}
\end{array}\right]_{l \times m}
$$

where the ' $\boldsymbol{\beta}$ ' is the network weight between the hidden layer and the output layer, and ' $\beta_{i j}$ ' is the weight between the $i^{\text {th }}$ hidden node of the hidden layer and the $j^{\text {th }}$ hidden node of the output layer. ' $m$ ' is the number of output nodes in output layer.

$$
b=\left[\begin{array}{llll}
b_{1} & b_{2} & \cdots & b_{l}
\end{array}\right]_{l \times 1}^{-1}
$$

where ' $b$ ' is the threshold of the hidden layer.

$X$ is supposed to be the input matrix and the history data $X$ are used to train the ELM network.

$$
\boldsymbol{X}=\left[\begin{array}{cccc}
x_{11} & x_{12} & \cdots & x_{1 p} \\
x_{21} & x_{22} & \cdots & x_{2 p} \\
\vdots & \vdots & & \vdots \\
x_{n 1} & x_{n 2} & \cdots & x_{n p}
\end{array}\right]_{n \times p}
$$

The real outputting matrix of the ELM network can be defined as below:

$$
\boldsymbol{T}=\left[\begin{array}{llll}
\boldsymbol{t}_{1} & \boldsymbol{t}_{2} & \cdots & \boldsymbol{t}_{p}
\end{array}\right]_{m \times p}
$$

And based on the equations (1)-(4), the real outputting matrix of the ELM can be defined as follow:

$$
\begin{aligned}
\boldsymbol{t}_{\boldsymbol{j}} & =\left[\begin{array}{c}
t_{1 j} \\
t_{2 j} \\
\vdots \\
t_{m j}
\end{array}\right]_{m \times 1} \\
& =\left[\begin{array}{c}
\sum_{i=1}^{l} \beta_{i 1} g\left(\boldsymbol{\omega}_{\boldsymbol{i}} \boldsymbol{X}_{\boldsymbol{j}}+b_{i}\right) \\
\sum_{i=1}^{l} \beta_{i 2} g\left(\boldsymbol{\omega}_{\boldsymbol{i}} \boldsymbol{X}_{\boldsymbol{j}}+b_{i}\right) \\
\vdots \\
\sum_{i=1}^{l} \beta_{i m} g\left(\boldsymbol{\omega}_{\boldsymbol{i}} \boldsymbol{X}_{\boldsymbol{j}}+b_{i}\right)
\end{array}\right]_{m \times 1},(j=1,2,3, \ldots \ldots, p)
\end{aligned}
$$

where

$$
\begin{aligned}
\boldsymbol{\omega}_{\boldsymbol{i}} & =\left[\begin{array}{llll}
\omega_{i 1} & \omega_{i 2} & \cdots & \omega_{i n}
\end{array}\right] \\
\boldsymbol{X}_{\boldsymbol{j}} & =\left[\begin{array}{llll}
x_{1 j} & x_{2 j} & \cdots & x_{n j}
\end{array}\right]^{T}
\end{aligned}
$$

$g(x)$ is an activation function in the hidden layer of the ELM.

The following equations can be acquired by Eqs. (5)-(8):

$$
\hat{\boldsymbol{\beta}}=\boldsymbol{H}^{-1} \boldsymbol{T}^{T}
$$

where

$$
\begin{aligned}
\boldsymbol{H} & \left(\boldsymbol{\omega}_{1}, \boldsymbol{\omega}_{2}, \cdots, \boldsymbol{\omega}_{l}, b_{1}, b_{2}, \cdots, b_{l}, \boldsymbol{X}_{1}, \boldsymbol{X}_{2}, \cdots \boldsymbol{X}_{p}\right) \\
= & {\left[\begin{array}{cccc}
g\left(\boldsymbol{\omega}_{1} \cdot \boldsymbol{X}_{1}+\boldsymbol{b}_{1}\right) & g\left(\boldsymbol{\omega}_{2} \cdot \boldsymbol{X}_{1}+b_{2}\right) & \cdots & g\left(\boldsymbol{\omega}_{l} \cdot \boldsymbol{X}_{1}+b_{l}\right) \\
g\left(\boldsymbol{\omega}_{1} \cdot \boldsymbol{X}_{2}+\boldsymbol{b}_{1}\right) & g\left(\boldsymbol{\omega}_{2} \cdot \boldsymbol{X}_{2}+b_{2}\right) & \cdots & g\left(\boldsymbol{\omega}_{l} \cdot \boldsymbol{X}_{2}+b_{l}\right) \\
\vdots & \vdots & \vdots & \vdots \\
g\left(\boldsymbol{\omega}_{1} \cdot \boldsymbol{X}_{p}+\boldsymbol{b}_{1}\right) & g\left(\boldsymbol{\omega}_{2} \cdot \boldsymbol{X}_{p}+b_{2}\right) & \cdots & g\left(\boldsymbol{\omega}_{l} \cdot \boldsymbol{X}_{p}+b_{l}\right)
\end{array}\right] }
\end{aligned}
$$

And $\boldsymbol{H}^{-1}$ is pseudo-inverse matrix of $\boldsymbol{H}$. The ELM can be solved in the following algorithm:

Algorithm ELM: Given a training set $\left\{(\boldsymbol{X}, \boldsymbol{T}) \mid \boldsymbol{X} \in \boldsymbol{R}^{n \times p}\right.$, $\boldsymbol{T} \in \boldsymbol{R}^{m \times p}$, activation function $g(x)$, testing set $\hat{\boldsymbol{X}}$ and hidden node number $p$.

Step one: Randomly assign input weight $\omega$ and bias $b$. Step two: Calculate the hidden layer output matrix $\boldsymbol{H}$. Step three: Calculate the output weight matrix $\boldsymbol{\beta}$.

Step four: Input matrix $\hat{X}$ and get the output testing results by the transform (9).

\section{Error correction}

Based on the ELM forecasting results, an error correction model is applied to obtain the ultra-shortterm forecasting. The persistence method is used as a benchmark model to examine whether an advanced model can perform well. In this model the future 
wind power will be the same as occurred in the present time step as given by

$$
\hat{P}_{t+k \mid t}=P_{t}
$$

where $\hat{P}_{t+k \mid t}$ is the forecast at time $t$ for the look-ahead time $k$ and $P_{t}$ is the measurement at time $t$.

In comparison with wind power, the temporal characteristics of wind power forecasting errors are less mentioned in literatures. However, it is found that the forecasting error level at next time point tends to keep the same as present time point by analyzing the states transition probability among different error levels. Thus, the error for next time point can be written as

$$
\hat{e}_{t+1 \mid t}=e_{t}
$$

where $e_{t}$ is the deviation between forecasted and measured wind power.

$$
e_{t}=p_{t}-\hat{p}_{t \mid t-1}
$$

The computed error is then added to the forecasted wind power for next time point to get the corrected forecasting.

$$
\tilde{p}_{t+1 \mid t}=\hat{p}_{t+1 \mid t}+\hat{e}_{t+1 \mid t}=\hat{p}_{t+1 \mid t}+e_{t}
$$

The flow chart of wind power forecasting procedure is shown in Fig. 2.

\section{Data description and pre-processing}

The proposed model is verified using the measured data in a wind farm located in the northern China for a period of about 15 months from 24 February 2014 to 31 May 2015. The 41072 non-consecutive data points before 02 March 2015 are used for training the ELM models whereas the consecutive time series of 66 days from 02 March 2015 to 31 May 2015 is used to verify the models performances. The total installed capacity of the wind farm is $50 \mathrm{MW}$. The measured data are used for both training the ELM model and verifying the model. The time scale of collecting data is $15 \mathrm{~min}$. The scatter of wind power versus wind speed of the wind farm is plotted in Fig. 3.

The characteristic of wind speed is shown as the frequency histogram in Fig. 4. It can be well fitted using a Webull distribution.

The mechanical power extracted from wind by a wind turbine is a function of the wind speed, blade pitch angle, and shaft speed. The algebraic equation shown below characterizes the power extracted [23].

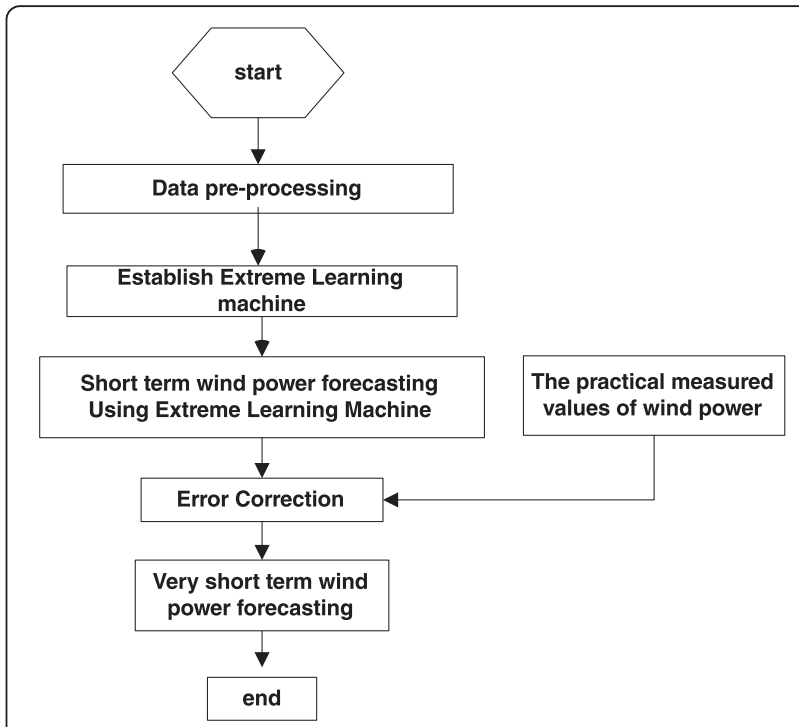

Fig. 2 Flow chart of wind power forecasting procedure

$$
P_{m}=\frac{1}{2} \rho v_{w}^{3} \pi r^{2} C_{p}(\lambda)
$$

where $P_{m}$ is the power extracted from the wind, in watts; $\rho$ the air density, in $\mathrm{kg} / \mathrm{m}^{3} ; r$ the radius swept by the rotor blades, in $\mathrm{m} ; v_{w}$ the wind speed, in $\mathrm{m} / \mathrm{s} ; C_{p}$ the performance coefficient; $\lambda$ the tip ratio, i.e., the ratio of turbine blade speed to that of the wind

$$
\lambda=\frac{\omega_{t} r}{v_{w}}
$$

where $\omega$ is mechanical rotor speed in radians/s.

From Eq. (15) it is noted that the air density, the wind speed are not quantities that can be controlled. That means a wind turbine will yield

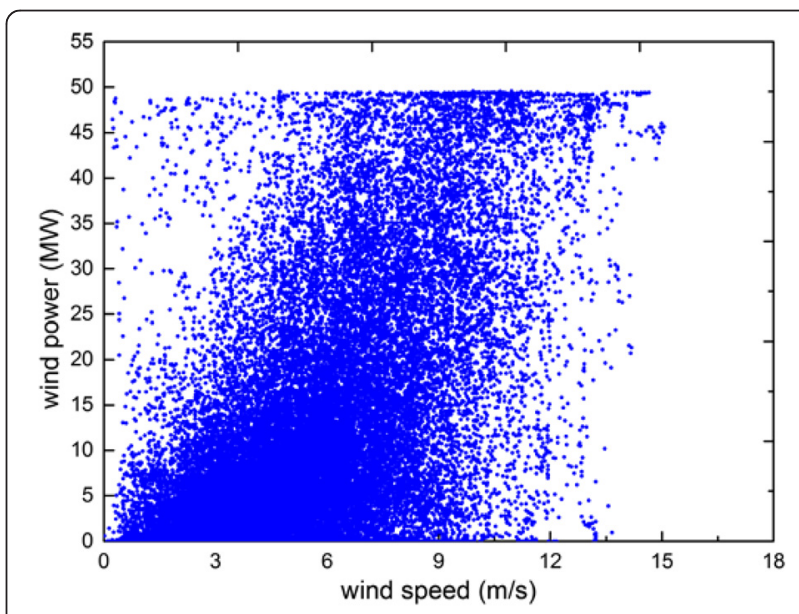

Fig. 3 Scatter plot of wind power versus wind speed 


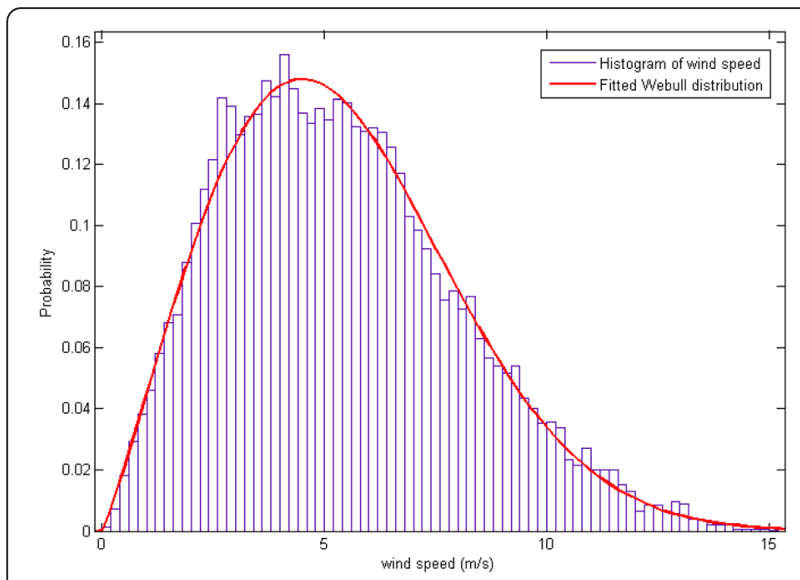

Fig. 4 Characteristics of wind speed data

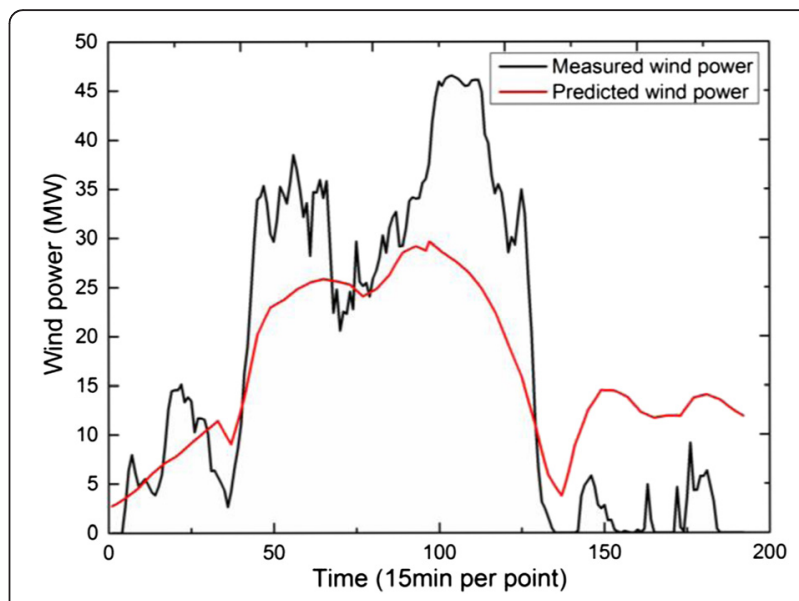

Fig. 6 Short-term forecasting results of ELM and their corresponding measurements

2) When the wind speed is close to zero but the wind farm output is very large (e.g. larger than half of the rated capacity of the wind farm).

Moreover, wind speed and power data are normalized by using the following formula

$$
x_{\text {normal }}=\frac{x-\max (x)}{\max (x)-\min (x)}
$$

anomalies are supposed to be eliminated before building an ELM model.

1) When the wind speed is very large (e.g. larger than $5 \mathrm{~m} / \mathrm{s}$ ) but the corresponding wind power is close to zero.

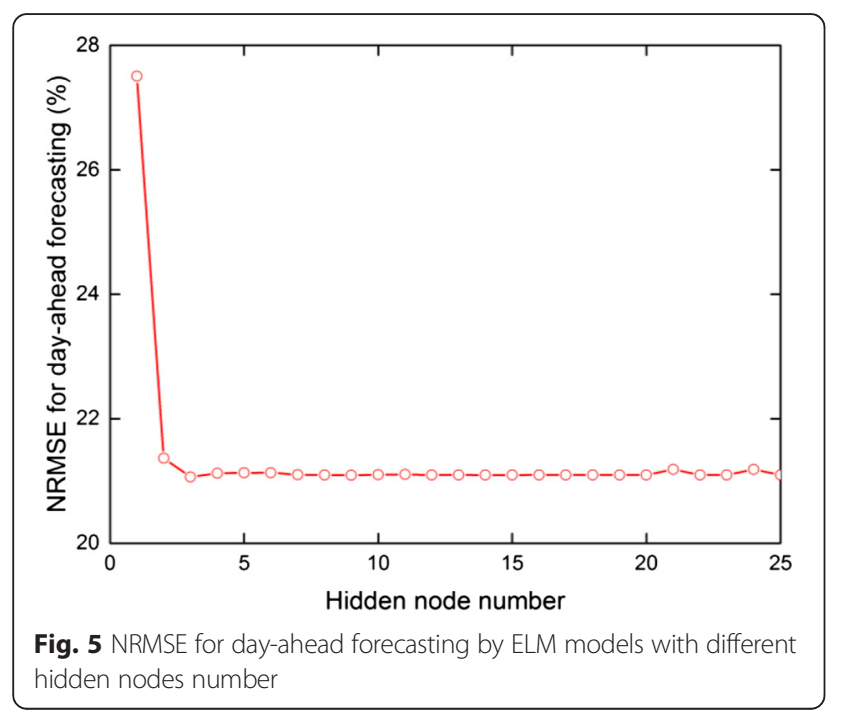

where $x$ is the original data, $x_{\text {normal }}$ is the normalized data, $\max (x)$ is the maximum of original datasets, and $\min (x)$ is the minimum of original datasets.

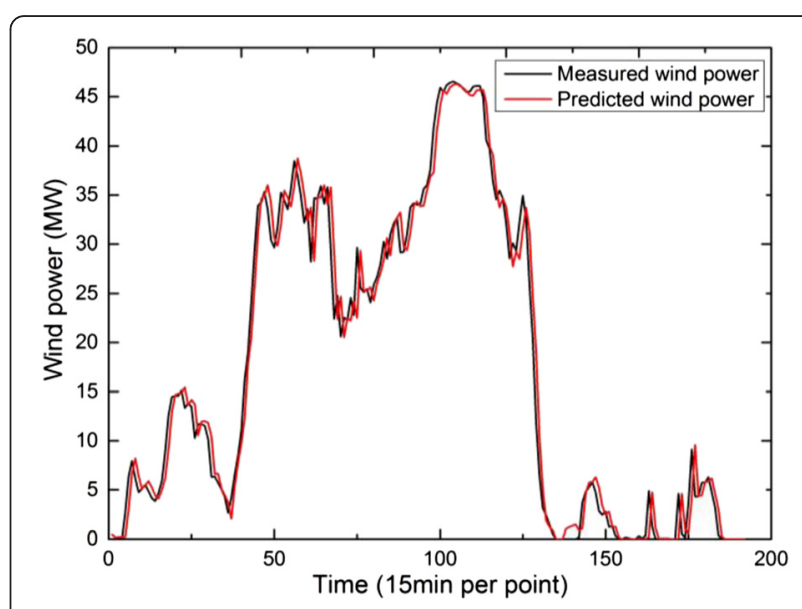

Fig. 7 Ultra-short-term forecasting results and their corresponding measurements after error correction 


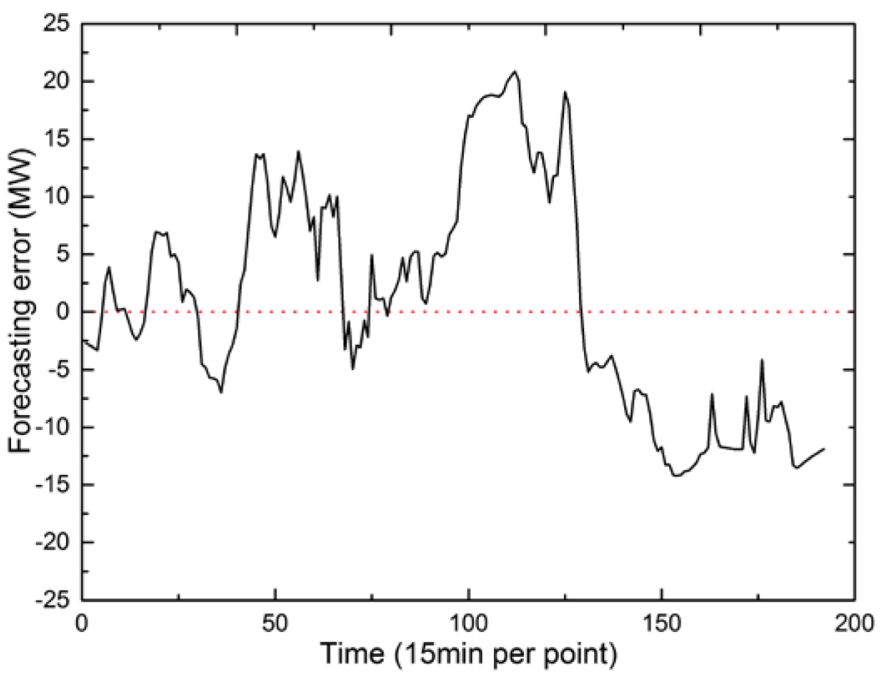

Fig. 8 Short-term forecasting error by using ELM

\section{Results and discussion Model parameterization}

The input of the ELM model is wind speed and the output is wind power, hence the number of nodes for both of input layer and output layer is set as one. As for the hidden layer, different values of the hidden nodes number are tested. The criterion for quantifying the performance of wind power forecasting is normalized root mean squared error (NRMSE).

$$
\text { NRMSE }=\frac{\sqrt{\sum_{i=1}^{n}\left(p_{i}-\hat{p}_{i}\right)^{2}}}{\operatorname{Cap} \cdot \sqrt{n}}
$$

NRMSE for day-ahead (24 h-ahead) forecasting by ELM models with different hidden nodes number are shown in Fig. 5.

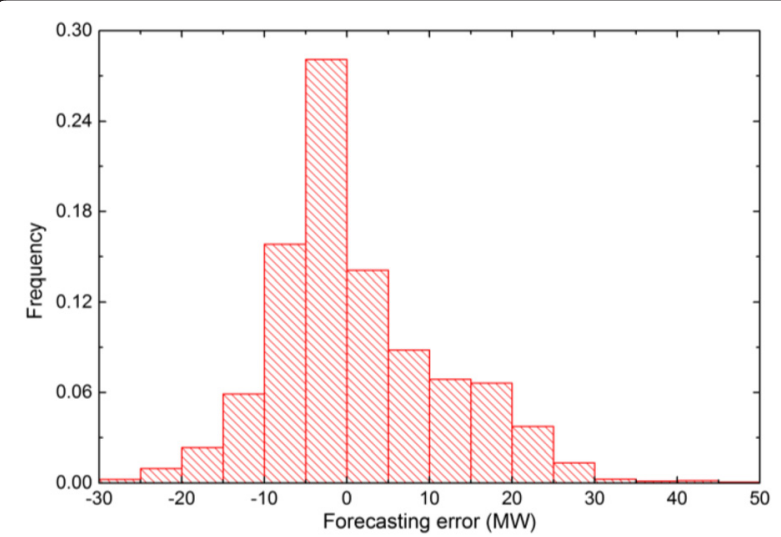

Fig. 9 Short-term forecasting error distribution by using ELM
From Fig. 5 it can be seen that, the value of NRMSE decreases dramatically when the hidden nodes number ranges from 1 to 3 . With 3 hidden nodes, the ELM has the best performance of $21.09 \%$ in terms of NRMSE. Adding more nodes will not lead to better results. Thus, the nodes number is finally set to be 3 .

\section{Results and analysis}

The day-ahead forecasting results in short-term scale before error correction by using ELM and their corresponding measurements dated from 22 April 2015 to 23 April 2015 are shown in Fig. 6. The 15 min-ahead forecasting results in ultra-short-term scale and their corresponding measurements after error correction are shown in Fig. 7.

In Fig. 6, the ELM doesn't perform well due to the strong stochastic feature of wind and the weak relationship between wind farm output and wind speed. Particularly, the ramp events are not accurately forecasted. It can be seen that large errors occur around peak value of measurement curve, where wind power changes drastically in a short time. The overall NRMSE of forecasting results for 66 days is $21.09 \%$.

The short-term forecasting errors are shown in Fig. 8 and their distribution is shown in Fig. 9. Ultrashort-term forecasting error after error correction is depicted in Fig. 10. As can be seen from Fig. 10, the forecasting error fluctuates drastically with large amplitude. The maximal error reaches up to $50 \%$ of installed capacity of the wind farm. It is noted that from Fig. 8, though most of the errors lie in the interval of [-20 MW, $20 \mathrm{MW}$, there is still a portion of large errors that cannot be neglected. It is indicated that the spread range of errors distribution is 


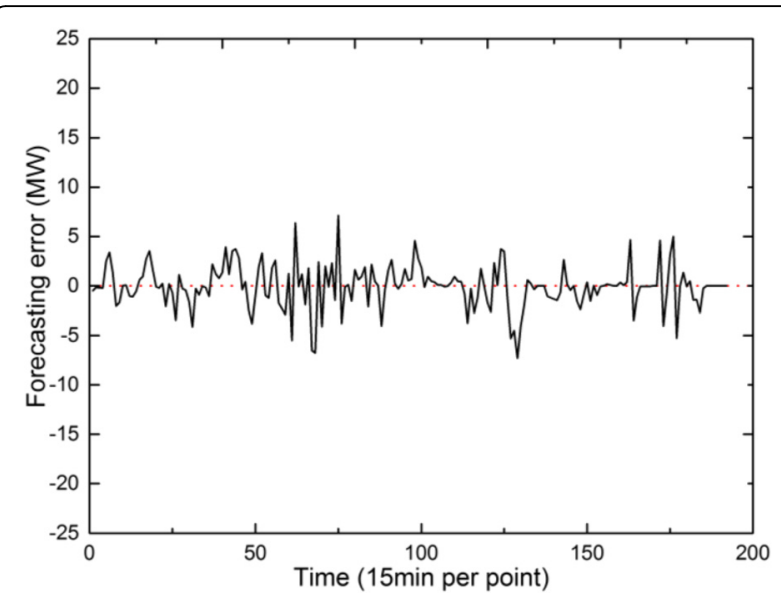

Fig. 10 Ultra-short-term forecasting error after error correction

large and positively biased, which reveals the poor forecasting performance.

While in Fig. 7 the ultra-short-term forecasting curve follows closely with the measurement curve. In addition, Figs. 10 and 11 show that most of forecasting errors lie in the interval of $[-10 \mathrm{MW}$, $10 \mathrm{MW}$. The distribution is concentrated and almost unbiased. The overall NRMSE is $5.76 \%$, indicating a good result of the correction method for ultra-shortterm forecasting.

In terms of the computational efficiency, time consumed for training and testing ELM models with varying hidden nodes number is depicted in Fig. 12.

It is noted that the computation time is approximately proportional to the hidden nodes number. When the hidden nodes number is 3 , the computation time is $1.5377 \mathrm{~s}$, including the time for training model with 41072 data points and forecasting 6336 data points. That indicates a high computational

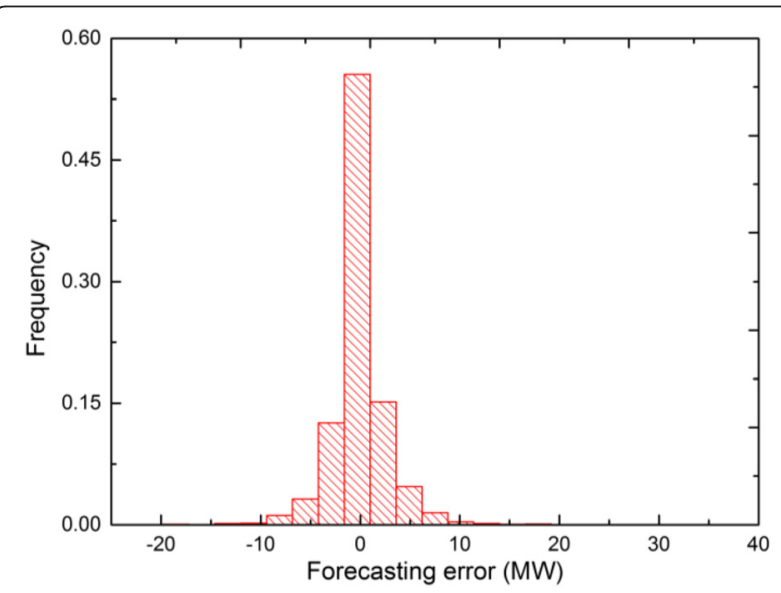

Fig. 11 Ultra-short-term forecasting error distribution after error correction

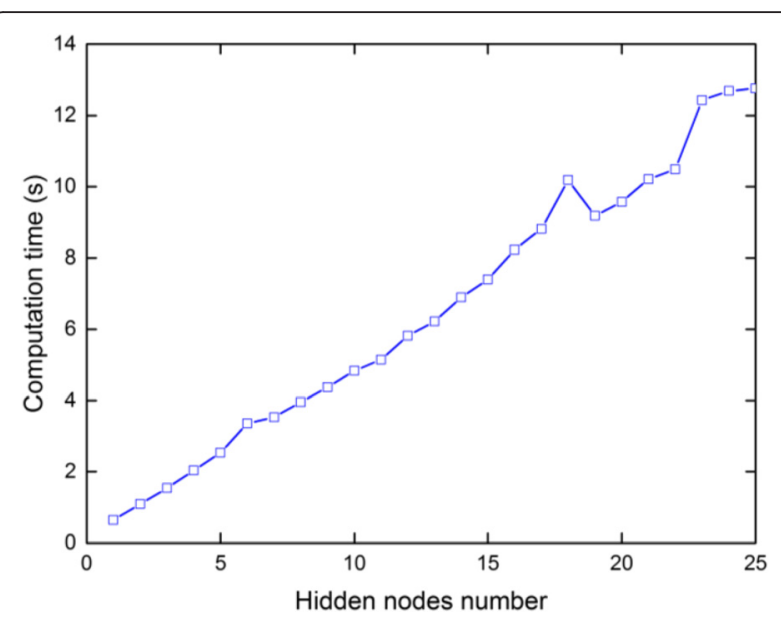

Fig. 12 Computation time for training and testing ELM models with varying hidden nodes number

efficiency for wind power forecasting, which can satisfy the practical needs.

\section{Conclusions}

In this paper, an extreme learning machine model with error correction with higher efficiency is developed to predict power output of a wind farm in a ultra-shortterm time scale. Case study shows that the ultra-shortterm wind forecasting accuracy is further improved in terms of normalized root mean squared error (NRMSE).

\section{About the Authors}

Zhi Li was born in Hubei, China, in 1994. She received her B.Sc. in applied mathematics from Department of Math, China Agricultural University in 2015. She is currently a PhD student of the Department of Electric Power Systems, China Agricultural University(CAU), Peoples Republic of China. Her research interests are wind power forecasting, model predictive control(MPC) in wind power operations.

Lin Ye (M'98) received his B.Sc. from WuHan University of Hydraulic \& Electric Engineering, Peoples Republic of China in 1992 and his Ph.D. degree in 2000 from the Institute of Electrical Engineering (IEE), Chinese Academy of

Sciences (CAS), all in electrical engineering. He has been pursuing research at ForschungsZentrum Karlsruhe(FZK) (*now merged with University of Karlsruhe $(\mathrm{TH})$ to form Karlsruhe Institute for Technology,KIT) as a research fellow of Alexander von Humboldt Stiftung/Foundation (AvH) of Germany from 2000 to 2002. In 2004, Dr. Lin Ye joined the Interdisciplinary Research Center (IRC) in Superconductivity, Department of Engineering/Cavendish Laboratory, The University of Cambridge, United Kingdom, as a research fellow. At Cambridge Laboratory, he had been involved in developing a novel resistive type of superconducting fault current limiter prototype for electrical marine propulsion which was funded by Rolls-Royce Plc and the Department of Trade \& Industry (DTI) of the United Kingdom. He received the "Hua Wei" Award from Chinese Academy of Sciences in 1999, and Research Young Investigator Award from Fok Ying Tung Education Foundation, Ministry of Education, Peoples Republic of China in 2003. He was an awardee of the Program for New Century Excellent Talents in China Universities in 2008. $\mathrm{He}$ is currently a full professor in electrical power engineering at the Department of Electric Power Systems, China Agricultural University (CAU), Beijing, Peoples Republic of China, where he has headed the Power System Off-line/Real Time Simulations Laboratory. Dr. Lin Ye holds memberships in IEEE (USA), and European EMTP-ATP Users Group (EEUG) as well as senior member of Wolfson College at Cambridge University. He is also a member of the IEEE Task Force on Sustainable Energy Systems for Developing Communities. His research interests are in the areas of electrical power 
system analysis \& control, Electromagnetic Transient Program (EMTP) modeling \& simulation, power applications of superconductivity, Renewable Energy Generation \& System Integration and Wind/Solar Power Forecasting. Yongning Zhao was born in Henan, China, in 1990. He received his B.Sc. in electrical engineering from China Agricultural University in 2012. He is currently a Ph.D. student of the Department of Electric Power Systems, China Agricultural University (CAU), Peoples Republic of China. His research interests are in the areas of power system operation and control, analysis of the temporal-spatial characteristics of wind power, wind power forecasting and integration.

Xuri Song was born in Weihai city, Shangdong Province, China, in 1987. He received his B.Sc. and M.Sc. in electrical engineering from China Agricultura University in 2009 and 2011 respectively. He is now an engineer with the Department of Electrical Power Automation, China Electric Power Research Institute(CEPRI). His research interests are power grid operation \& control, power grid state estimation with high penetration of renewables.(email:songxuri@epri.sgcc.com.cn).

Jingzhu Teng was born in Beijing, China, in 1991. She received her B.Sc. with Renewable Energy Generation from North China Electric Power University, Beijing, Peoples Republic of China in 2013. She has been working as an engineer in Beijing Energy Services Company of State Grid for two years. She is currently a M.Sc. student of the Department of Electric Power Systems, China Agricultural University (CAU), Peoples Republic of China. Her research interest is wind power forecasting

Jingxin Jin was born in Hohhot City, Inner Mongolia, China, in 1984. He received his Master of Engineering in electrical engineering from China Agricultural University in 2014. He is now an engineer and project manager with the Inner Mongolia Water Resources and Hydropower Survey and Design Institute, Hohhot, Inner Mongolia 010021, China His research interests are wind farm micrositing, wind power forecasting and wind resources assessment.(Email: intel-10@163.com).

\section{Acknowledgment}

This work is supported by National Natural Science Foundation of China under grants 51477174 and 51077126 , the Key Project of Chinese Ministry of Education under Grant 109017. The authors also acknowledge Specialized Research Fund for the Doctoral Program of Higher Education of China under contract 20110008110042 and the support from China Electric Power Research Institute under contract DZB51201503568.

\section{Authors' contributions}

ZL carried out the short-term wind power forecasting and error correction and participated in the design of the study. LY conceived of the study and participated in its design and coordination. $Y Z$ participated in the design of the study, statistical analysis and drafting the manuscript. XS participated in the statistical analysis. JT participated in the statistical analysis. JJ participated in drafting the manuscript. All authors read and approved the final manuscript.

\section{Competing interests}

The authors declare that they have no competing interests.

\section{Author details}

${ }^{1}$ College of Information and Electrical Engineering, China Agricultural University, P. O. Box 210, Beijing 100083, Peoples Republic of China. ${ }^{2}$ Department of Electric Power Automation, China Electric Power Research Institute, Beijing 100192, China. ${ }^{3}$ Inner Mongolia Water Resources and Hydropower Survey and Design Institute, Hohhot, Inner Mongolia 010021 , China.

\section{Received: 10 May 2016 Accepted: 10 May 2016}

\section{Published online: 20 June 2016}

\section{References}

1. Juban, J, Sibert, N, Kariniotakis, GN (2007). Probabilistic short-term wind power forecasting for the optimal management of wind generation (pp. 683-688). Lausanne: IEEE Powertech.

2. Giebel, G, Brownsword, R, Kariniotakis, G, Denhard, M, Draxl, C. The state of the art in short-term prediction of wind power a literature overview. Deliverable Report of the Anemos Project, 2011; Available from: http://orbit. dtu.dk/en/publications/the-stateoftheart-in-shortterm-prediction-of-windpower(0d76e147-4bfc-444b-af00-13a0db8f132e).html. Accessed 19 May 2016.

3. Deob, MC, Ghosh, S, Kulkarnia, S. Effect of Climate Change on Wind Persistence at Selected Indian Offshore Locations. 8th International Conference on Asian and Pacific Coasts (APAC 2015): Indian Institute of Technology Madras. Volume 116. pp. 615-622. http://www.apac2015.com/

4. Agüera-Pérez, A, Palomares-Salas, JC, González de laRosa, JJ, Moreno-Muñoz, A (2013). Spatial persistence in wind analysis. Journal of Wind Engineering and Industrial Aerodynamics, 119, 48-52.

5. Sideratos, G, Hatziargyriou, ND. (2007). An advanced statistical method for wind power forecasting. IEEE Transactions on Power Systems, 22(1), 258-265.

6. Tascikaraoglu, A, Uzunoglu, M. (2014). A review of combined approaches for prediction of short-term wind speed and power[J]. Renewable and Sustainable Energy Reviews, 34, 243-254.

7. Jung, J, Broadwater, RP. (2014). Current status and future advances for wind speed and power forecasting[J]. Renewable and Sustainable Energy Reviews, 31, 762-777.

8. Erdem, E, Shi, J. (2011). ARMA based approaches for forecasting the tuple of wind speed and direction[J]. Applied Energy, 88(4), 1405-1414.

9. Cao, Q, Ewing, BT, Thompson, MA. (2012). Forecasting wind speed with recurrent neural networks[J]. European Journal of Operational Research, 221(1), 148-154.

10. Li, LI, Lin, YE. (2011). Identification of singular points in wind speed data. Power Syst Prot Control, 39(22), 92-97 (in Chinese).

11. Barbounis, TG, Theocharis, JB, Alexiadis, MC, Dokopoulos, PS. (2006). Longterm wind speed and power forecasting using local recurrent neural network models. IEEE Transactions on Energy Conversion, 21(1), 273-284.

12. Catalao, JPS, Pousinho, HMI Mendes, VMF. (2009). An artificial neural network approach for short-term wind power forecasting in Portugal. In Intelligent System Applications to Power Systems (ISAP) International Conference (pp. 1-5).

13. $\mathrm{Hu}, \mathrm{Q}, \mathrm{Zhang}, \mathrm{R}$, Zhou, Y. (2016). Transfer learning for short-term wind speed prediction with deep neural networks[J]. Renewable Energy, 85, 83-95.

14. Dangar, PB, Kaware, SH, Katti, PK. (2010). Offshore wind speed forecasting by SVM and power integration through HVDC light. In IPEC Conference Proceedings (pp. 962-967).

15. Lin, YE, Peng, L. (2011). Combined model based on EMD-SVM for short-term wind power prediction. Proceedings of the Chinese Society for Electrical Engineering (CSEE), 31(11), 102-108 (in Chinese).

16. Madsen, H, Nielsen, HA, Nielsen, TS. (2005). A tool for predicting the wind power production of off-shore wind plants. In Copenhagen Offshore Wind Conference \& Exhibition. Copenhagen: Danish Wind Industry Association.

17. Nielsen, TS, Madsen, H, Nielsen, HA, Giebel, G, Landberg, L. (2001). Zephyr - the prediction models. Copenhagen: European Wind Energy Conference.

18. Rodríguez-García, JM, Domínguez, T, Alonso, JF, Imaz, L. (2007). Large intergration of wind power: the Spanish experience. Tampa: PES General Meeting

19. Zack, J, Bailey, B, Brower, M. Wind energy forecasting: the economic benefits of accuracy. In: Wind Power Asia 2006 July 2010; Available from: https:// www.researchgate.net/publication/228681909_Wind_Energy_Forecasting_ The_Economic_Benefits_of_Accuracy. Accessed 19 May 2016.

20. Monteiro, C, Bessa, R, Miranda, V, et al. Wind power forecasting: state-of-theart 2009[R]. Argonne National Laboratory (ANL), 2009; Available from: http:// www.osti.gov/scitech/biblio/968212. Accessed 19 May 2016.

21. Huang, GB, Zhu, QY, Siew, CK. (2006). Extreme learning machine: theory and applications[J]. Neurocomputing, 70(1), 489-501.

22. Liu, H, Tian, H, Li, Y. (2015). Four wind speed multi-step forecasting models using extreme learning machines and signal decomposing algorithms[J]. Energy Conversion and Management, 100, 16-22.

23. Vittal, V, Ayyanar, R. (2013). Grid Integration and Dynamic Impact of Wind Energy. New York: Springer. 